\title{
A Composite Material Leaf Spring with Fiber Bragg Grating as a Sensor System for Dynamic Vehicle Loading
}

\author{
Tso-Sheng Hsieh, Chia-Chin Chiang* and Chia-Hao Lin \\ Department of Mechanical Engineering, National Kaohsiung University of Applied Sciences, \\ 415 Chien Kung Road, Kaohsiung 807, Taiwan
}

(Received December 24, 2013; accepted March 6, 2014)

Key words: $\quad$ weigh-in-motion, composite material, leaf spring, fiber Bragg grating

A fiber Bragg grating sensor attached to the composite material leaf spring as a sensor system is proposed for dynamic vehicle loading. The load borne by the leaf spring was calculated from the period change of the fiber grating caused by the deformation of the composite material leaf spring under loading. From the results of a light intensity calibration experiment, a linear relationship between load and voltage signal was found when the composite material leaf spring was deformed under loading. The R-squared value of the linear relationship reached up to 0.984. Data from a temperature compensation experiment supported the reliability of the temperature compensation framework of the study.

\section{Introduction}

Recently, it has become more difficult to maintain roads owing to increasingly heavier overweight trucks used for transportation, and the current weigh-in-motion (WIM) system has problems retrieving data regarding overweight vehicles on highways. ${ }^{(1)}$ The weighing apparatus now uses strain gauge ${ }^{(2)}$ sensors as the vehicle load weighing system. A strain gauge system has a very short lifetime as the strain gauge can be easily damaged by weather or human error. As such, there is a continuing need for improved sensors that can accurately determine the weight of moving trucks.

Fiber Bragg gratings (FBGs) have been used to sense a number of physical parameters, including vibrations, temperatures, and strains, and in structure health monitoring. ${ }^{(3,4)}$ An FBG-based WIM system using fiber-reinforced composite laminates as the load-supporting material has shown good repeatability and high linearity under static testing. ${ }^{(5)}$ FBG sensors are sensitive to both strain and temperature. Therefore, to obtain the strain value, a second FBG must be used for temperature compensation. ${ }^{(6)}$ The purpose of this work was to use FBG attached to a composite material leaf spring

*Corresponding author: e-mail: ccchiang@kuas.edu.tw 
for load measurement and temperature compensation. We aimed to establish a dynamic sensing system for measuring the load of heavy vehicles and to promote the sensing technology for vehicle load measurement.

\section{Fundamental Principles of Fiber Bragg Grating}

FBG consists of thousands of short-period refractive index modulations. FBG has a grating period of approximately $1 \mu \mathrm{m}$. When a broadband light source illuminates the FBG, a certain wavelength of the light will be reflected. The reflected FBG wavelength can be expressed as ${ }^{(7)}$

$$
\lambda_{\mathrm{B}}=2 n_{\mathrm{eff}} \Lambda,
$$

where $\lambda_{\mathrm{B}}$ is the Bragg wavelength, $n_{\mathrm{eff}}$ is the effective refractive index, and $\Lambda$ is the grating period. When the grating zone is under strain, the wavelength will drift.

\section{Experiment}

\subsection{Static experiment and light intensity calibration experiment}

FBG sensor A $(1552.52 \mathrm{~nm})$ and sensor B $(1545.48 \mathrm{~nm})$ were fused in series for use in the experiment. Furthermore, a corrugated long-period fiber grating (CLPFG) filter (resonance wavelength at $1548.4 \mathrm{~nm}$ ) was fused in a series to filter the wave for the repeated recording of experiment results. The framework is illustrated in Fig. 1. Sensor A was attached in the axial direction along the surface of the composite material leaf spring. Sensor B was used for temperature compensation. In addition, bearings

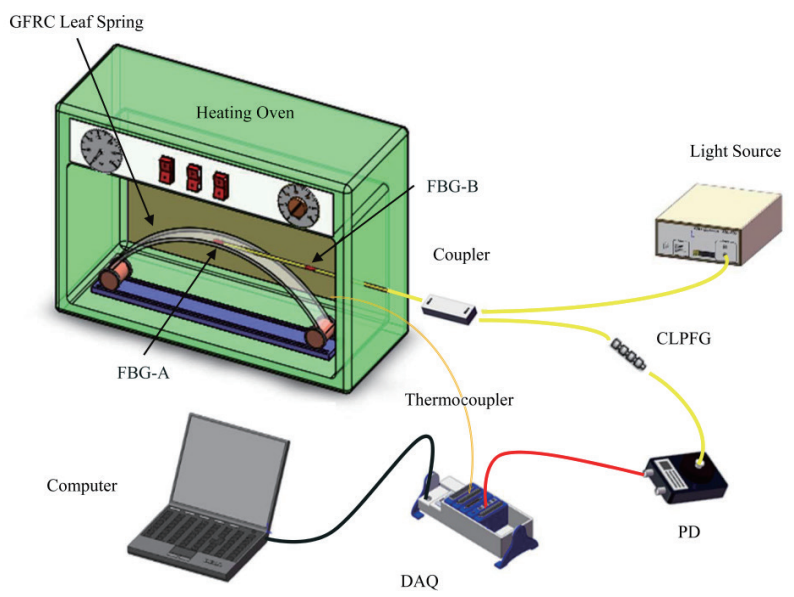

Fig. 1. (Color online) Schematic diagram for temperature compensation experiments. 
were added to the two ends of the composite material leaf spring. These bearings were placed on a smooth track to ensure that the composite material leaf spring could glide back and forth along the fixed track when the composite material leaf spring moved vertically under loading, thereby minimizing sliding friction. In addition, a broadband light ray was injected using an amplified spontaneous emission (ASE) broadband light source. The reflected spectra of the two FBG sensors were scanned and stored via an optical spectrum analyzer (OSA). The load was gradually increased every $0.8 \mathrm{~N}$ up to 7.2 $\mathrm{N}$ with a loading speed of $0.5 \mathrm{~mm} / \mathrm{min}$. The reflection spectrum recorded by the FBG sensors was recorded under every loading.

A photodetector was used to measure light intensity that is transformed into voltage with amplification. The voltage signal was acquired with the LabVIEW program.

\subsection{Temperature compensation experiment}

The setup of temperature compensation experiments is shown in Fig. 1. Under fixed loading conditions, a heating oven was used to heat up two FBG sensors simultaneously. The heating ranged from room temperature (approximately $30{ }^{\circ} \mathrm{C}$ ) to $70{ }^{\circ} \mathrm{C}$. Five heating experiments were conducted when the composite material leaf spring was loaded under $0,0.51,0.96,1.59$, and $2.16 \mathrm{~N}$. The measurement method used an ASE broadband light source to inject broadband light, which was reflected through two FBG sensors and then filtered with the CLPFG filter. An optical power amplifier was used to measure light intensity that is transformed into voltage with amplification. The voltage signal and temperature of the thermocouple were recorded using a data acquisition system.

\section{Results and Discussion}

\subsection{Static experiment and light intensity calibration experiment}

We first conducted an experiment on the relationship between loading and drift in the FBG sensor wavelength. Figure 2 shows the experiment results for FBG-A. The figure shows a good linear relationship between loading and drift of the Bragg wavelength. The R-squared value for the linear regression became to 0.991. Therefore, loading can be calculated from the measured drift of the FBG wavelength.

For a reflected light spectrum passing through the CLPFG, light intensity was filtered via the CLPFG in accordance with the Bragg wavelength under different loadings; that is, the intensity changed owing to the drift of the Bragg wavelength. In this experiment, the Bragg wavelength of the FBG-A sensor was located on the right side of the CLPFG resonance attenuation dip. Energy increased gradually because the Bragg wavelength drifted toward an elongated wavelength under loading with tensile strain. The energy increase under the CLPFG influence was linear with an R-squared value as high as 0.997.

When an FBG-B sensor was used for temperature compensation, there was no Bragg wavelength drift, as shown in Fig. 3. This is because FBG-B was not affected by tensile strain, and because the difference in room temperature was not significant. For the reflected light spectrum passing through the CLPFG, there was no significant difference in the light intensity under different loadings because of the lack of Bragg wavelength drift. 

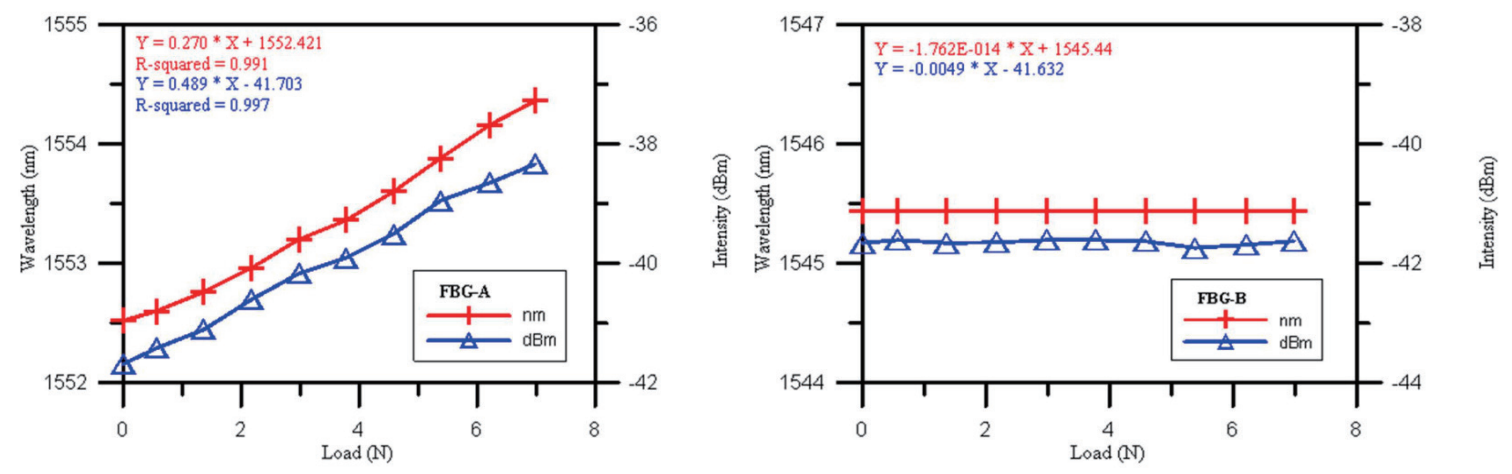

Fig. 2 (left). (Color online) Relationships among FBG-A loading, wavelength drift, and intensity of reflected light.

Fig. 3 (right). (Color online) Relationships among FBG-B loading, wavelength drift, and intensity of reflected light.

In Fig. 4, a linear relationship can be observed between loading and the displacement of the composite material leaf spring. The R-squared value for the linear relationship was as high as 0.985 . Therefore, the actual loading weight can be calculated from the amount of displacement of the composite material leaf spring. Data from the static experiment shows that there is a linear relationship between the wavelength drift and the applied load for the FBG sensor attached to the composite material leaf spring (Fig. 2). In addition, the light intensity of the reflected Bragg wavelength under different loads can decrease or increase linearly through the CLPFG. Therefore, with a constant temperature, the experiment applied load only to a composite material leaf spring in order to induce drift of the Bragg wavelength of the FBG sensor (FBG-A) attached to the composite material leaf spring. The light intensity of the reflected Bragg wavelength was further filtered by the CLPFG. An optical power amplifier was used to transform light into voltage with amplification and to measure the light intensity. Results shown in Fig. 4 indicate that there is a good linear relationship between load and voltage with an R-squared value as high as 0.984 . Therefore, we can adopt this framework and place it in the dynamic sensing system used in this study. The load exerted by a vehicle onto the weighbridge can be calculated from the measured voltage change caused by the displacement of the composite material leaf spring under load.

\subsection{Temperature compensation experiment}

When a leaf spring is not under loading and the temperature of the work environment changes, the temperature of the FGB-A attached to the composite material leaf spring and the temperature of the FBG-B sensor used for temperature compensation both change owing to temperature influence. In this experimental framework, the wavelength of the FBG-A sensor attached to the composite material leaf spring was located on the right side of the CLPFG resonance attenuation dip, and the wavelength of the FBG-B 

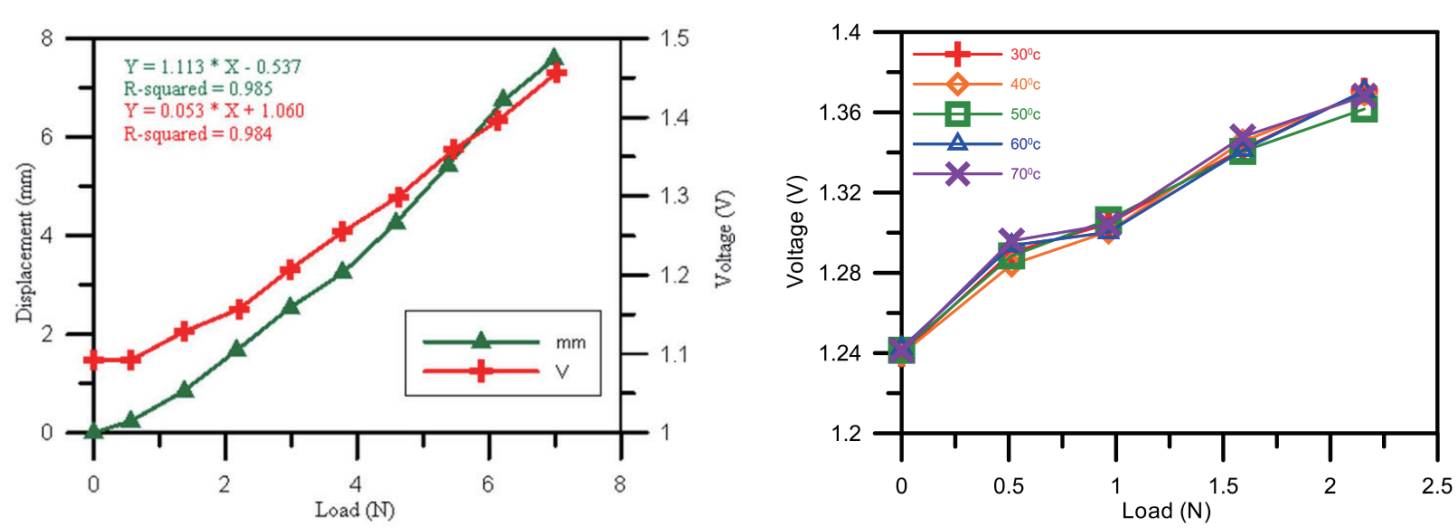

Fig. 4 (left). (Color online) Relationships among loading, displacement, and voltage changes.

Fig. 5 (right). (Color online) Voltage change when temperature is increased with loading.

sensor used for temperature compensation was located on the left side of the CLPFG resonance attenuation dip. Therefore, when the temperature in the work environment increases, the wavelengths of both FBG-A and FBG-B sensors increase (redshift). The light intensity gradually increases for FBG-A with the CLPFG filter, but it decreases for FBG-B with the CLPFG filter. When the temperature of the work environment decreases, the opposite results are obtained to achieve the temperature compensation effect. When the composite material leaf spring is not under different temperatures, loading was applied to a composite material leaf spring, the results of which are shown in Fig. 5. In this experiment, it was shown that the voltage change is very close under different temperatures when a composite material leaf spring is loaded. The results further validate the feasibility of the temperature compensation framework.

\section{Conclusions}

From the static experiment, we found a linear relationship between the wavelength of the FBG-A sensor and loading when there was deformation of the composite material leaf spring due to loading. When another FBG sensor was used for temperature compensation, there was no obvious change in the wavelength of the reflected light and light intensity of the reflected wavelength because of the lack of change in strain and temperature. In the light intensity calibration experiment, we used an optical power amplifier to transform the light intensity of the reflected wavelength from two FBG sensors through the CLPFG into voltage signals. We found a linear relationship between loading and voltage signal when there was deformation of the composite material leaf spring under loading. The R-squared value of the linear regression was as high as 0.984 . The data from the temperature compensation experiment supports the feasibility of the temperature compensation framework used in this study. 


\section{Acknowledgements}

This work was supported by the National Science Council under contract no. NSC 100-2628-E-151-002-MY3. We are also indebted to Professor S. K. Liaw of NTUST for the FBGs and equipment support.

\section{References}

1 J. F. Wang and M. G. Wu: Proc. Fifth World Congress on Intelligent Control and Automation, 2004, p. 5241.

2 S. K. Yang, T. S. Liu and Y. C. Cheng: Measurement 41 (2008) 491.

3 K. O. Hill and G. Meltz: J. Lightwave Technol. 15 (1997) 1263.

4 C. C. Ciang, J. R. Lee and H. J. Bang: Meas. Sci. Technol. 19 (2008) 122001.

5 K. Wang, Z. X. Wei, H. T. Zhang, X. Y. Huang, B. Q. Chen and H. L. Cui: Opt. Eng. 45 (2006) 064401 .

6 D. P. Zhou, L. Wei, W. K. Liu and J. W. Y. Lit: IEEE Photonics Technol. Lett. 21 (2009) 468.

7 T. Erdogan: J. Lightwave Technol. 15 (1997) 1277. 